\title{
Comparative study on aerodynamic heating under perfect and nonequilibrium hypersonic flows
}

\author{
Qiu Wang, JinPing Li* , Wei Zhao, and ZongLin Jiang \\ State Key Laboratory of High Temperature Gas Dynamics, Institute of Mechanics, Chinese Academy of Sciences, Beijing 100190, China
}

Received April 8, 2015; accepted June 4, 2015

\begin{abstract}
In this study, comparative heat flux measurements for a sharp cone model were conducted by utilizing a high enthalpy shock tunnel JF-10 and a large-scale shock tunnel JF-12, responsible for providing nonequilibrium and perfect gas flows, respectively. Experiments were performed at the Key Laboratory of High Temperature Gas Dynamics (LHD), Institute of Mechanics, Chinese Academy of Sciences. Corresponding numerical simulations were also conducted in effort to better understand the phenomena accompanying in these experiments. By assessing the consistency and accuracy of all the data gathered during this study, a detailed comparison of sharp cone heat transfer under a totally different kind of freestream conditions was build and analyzed. One specific parameter, defined as the product of the Stanton number and the square root of the Reynold number, was found to be more characteristic for the aerodynamic heating phenomena encountered in hypersonic flight. Adequate use of said parameter practically eliminates the variability caused by the deferent flow conditions, regardless of whether the flow is in dissociation or the boundary condition is catalytic. Essentially, the parameter identified in this study reduces the amount of ground experimental data necessary and eases data extrapolation to flight.
\end{abstract}

aerodynamic heating, hypersonic, shock tunnel, Stanton number, sharp cone

PACS number(s): 44.20.+b, 47.40.Ki, 07.35+k

Citation: Q. Wang, J. P. Li, W. Zhao, and Z. L. Jiang, Comparative study on aerodynamic heating under perfect and nonequilibrium hypersonic flows, Sci. China-Phys. Mech. Astron. 59, 624701 (2016), doi: 10.1007/s11433-015-5708-1

\section{Introduction}

The laws of hypersonic flow are critical to the successful design of hypersonic aerospace vehicles. High speed, shock compression, and viscous energy dissipation behind the bow shock of an aircraft head result in very high temperature. A massive amount of kinetic energy is then converted into heat energy, which makes the thermal environment become serous. To this effect, accurately predicting of the heat transfer rates is a key issue for researchers and developers working within the current space program.

Due to the high cost of flight tests, most aerodynamic heating experiments are completed in ground facilities,

*Corresponding author (email: lijinping@imech.ac.cn) where shock tunnels show their advantages for the accommodation of relatively large-size models and low operational costs. Advancements in experimental techniques have made it possible to realize hypersonic flows ranging from 2.5 to $45 \mathrm{MJ} / \mathrm{kg}$, which corresponds to velocities from 2 to $10 \mathrm{~km} / \mathrm{s}$ [1,2]. However, similarity parameters, such as Reynolds number or Mach number, differ from each other in different facilities due to their capability difference, making universal analysis, extrapolation, comparison, or adequate use of experimental data rather difficult [3]. Therefore, a data base and set of relevant principles obtained from original models remain highly necessary in order to build effective comparison between different ground facilities and facilitate successful ground-to-flight extrapolation. Cones have been the object of numerous experi- 
mental and computational investigations for their relative simplicity of the flowfields and widespread use in missile designs [4-9]. Bertin [10] tried to develop a data base for the calibration and validation of hypersonic CFD codes using sharp cones, but the base of information was still insufficient to meet the requirements and high-precision aerodynamic heating experimental data was limited, particularly under conditions where thermal chemical nonequilibrium prevailed. At the same time, with remarkable advances in physical models and computing methods, contributions of CFD on hypersonic flow simulation make great progress in the past two decades [11]. Experimental data with corresponding numerical analysis can give a better understanding of what we studied.

In the view of enthalpy and pressure requirements for hypersonic ground tests, the shock tunnel must incorporate a high performance driver. Among the existing driving techniques, the detonation drivers, in particular, are capable of producing high enthalpy and high pressure test flows simultaneously while being easily operated and requiring low capital investment [12]. In recent years, the backward and forward modes have been studied at LHD and other crucial techniques, such as spontaneous strong ignition. Attenuation of reflected waves has also been resolved successively [13]. As a result of these notable developments, a high enthalpy shock tunnel, JF-10, was constructed in 1996 followed by a large-scale shock tunnel, JF-12, in 2012, both of which have proven to be highly valuable tools for investigating into fundamental physics in hypersonic and high temperature gas flows $[14,15]$.

In this study, heat transfer measurements of a spherical sharp cone (7 deg half-angle) were conducted in both JF-10 and JF-12 shock tunnels, in which nonequilibrium and perfect gas flows were duplicated, respectively. Surface temperature was measured using home-made thermocouples and numerical analysis using the CFD technique was also conducted. The measurement precision of the thermocouples was discussed below in detail, followed by a comparison of nondimensional heat flux distributions along the body surface, aiming to find a preferable parameter for the comparison of experimental data in different ground facilities. The effects of gas state and boundary conditions on the parameter were also taken into consideration.

\section{Experimental facilities and test model}

The experimental program was conducted in the JF-10 and
JF-12 shock tunnels, both reflected shock tunnels using the detonation driving technique. A schematic of a detonation driven shock tunnel is shown in Figure 1. It consists of a damping section, detonation chamber, shock tube, nozzle and test section. A heavy metal diaphragm separates the detonation chamber and shock tube. The chamber is filled with a gaseous reactive mixture, typically oxygen and hydrogen. Then strong incident shock waves in the shock tube are easily generated by detonation product gases at high temperatures and pressures after simultaneously igniting the reactive mixtures. A detonation driven shock tunnel can be operated either in the backward mode to achieve longer driving time at a relatively low enthalpy level, such as JF-12, or in the forward mode to obtain the high enthalpy flow, such as JF-10. JF-10 is a high enthalpy shock tunnel able to provide the high temperature gas conditions for hypersonic flight and real gas effect can also be studied. However, JF-12 is the largest shock tunnel in the world, with the nozzle exit diameter of $2.5 \mathrm{~m}$, capable of replicating flight conditions for Ma5 9 at altitudes of $25-50 \mathrm{~km}$ to simulate integrated vehicle/engine conditions. The major specifications of the two shock tunnels are shown in Table 1 and other details are available in literature [14,15].

A relatively simple model configuration was selected in this study in order to minimize any uncertainties originating from geometric complexity. The model, a $7^{\circ}$ half-angle spherically sharp cone, is shown in in Figure 2. Considering the nozzle exit diameter of the two shock tunnels, a model with overall length of $590 \mathrm{~mm}$ was chosen for the JF-10 shock tunnel, and $1100 \mathrm{~mm}$ for the JF-12 shock tunnel. On each model, $1.4 \mathrm{~mm}$-diameter E-type (chromel-constantan) coaxial thermocouples, favored for rapid response time and ability to be flush-mounted, were installed along three generatrices to measure surface temperature. The number of thermocouples installed was determined by the space available inside the model. A typical temperature trace in JF-10 was shown in Figure 3. From the measured surface temperature $T$, the heat flux $\dot{q}$ was calculated according to Schults and Jones [16] as follows:

$$
\dot{q}\left(t_{n}\right)=2 \sqrt{\frac{\rho c k}{\pi}} \sum_{i=1}^{n} \frac{T\left(t_{i}\right)-T\left(t_{i-1}\right)}{\sqrt{t_{n}-t_{i}}-\sqrt{t_{n}-t_{i-1}}},
$$

where $\rho, c$, and $k$ are the density, heat capacity, and heat conductivity of the sensor material, respectively, $T$ and $t$ are temperature and time.

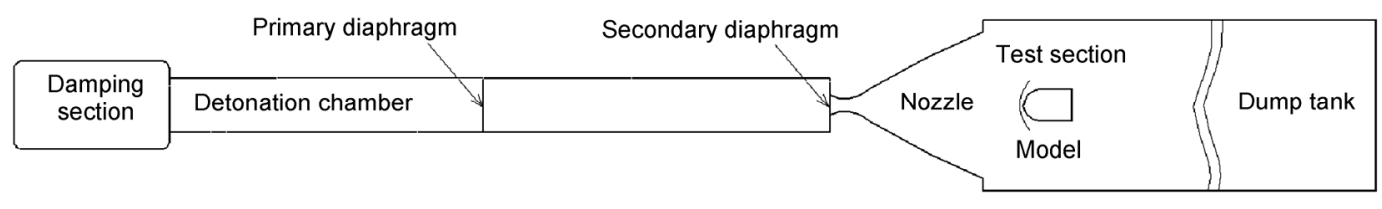

Figure 1 Detonation shock tunnel operation concept. 
Table 1 Facility comparison

\begin{tabular}{ccc}
\hline Facility & JF-10 & JF-12 \\
\hline Detonation & $10 \mathrm{~m}$ in length, & $100 \mathrm{~m}$ in length, \\
chamber & $150 \mathrm{~mm}$ in diameter & $400 \mathrm{~mm}$ in diameter \\
Shock tube & $12.5 \mathrm{~m}$ in length, & $88 \mathrm{~m}$ in length, \\
Operation mode & $100 \mathrm{~mm}$ in diameter & $720 \mathrm{~mm}$ in diameter \\
& forward mode & backward mode \\
Nozzle & conical & contoured \\
& $2 \mathrm{~m}$ in length, & $15 \mathrm{~m}$ in length, \\
Maximum $H_{0}$ & $0.5 \mathrm{~m} \mathrm{in} \mathrm{exit} \mathrm{diameter}$ & $2.5 \mathrm{~m} \mathrm{in} \mathrm{exit} \mathrm{diameter}$ \\
& $20 \mathrm{MJ} / \mathrm{kg}$ & $5 \mathrm{MJ} / \mathrm{kg}$ \\
\hline
\end{tabular}

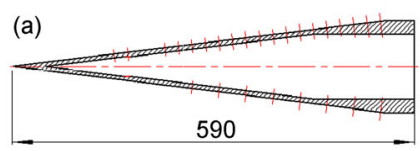

(b)

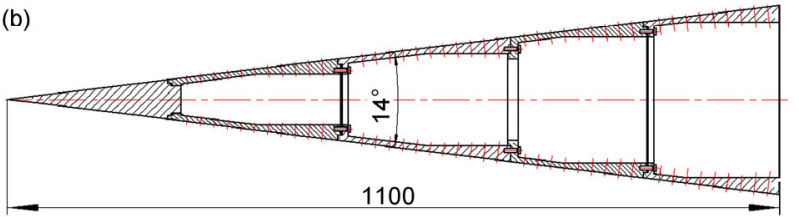

Figure 2 (Color online) Experimental models. (a) Model in JF-10; (b) model in JF-12.

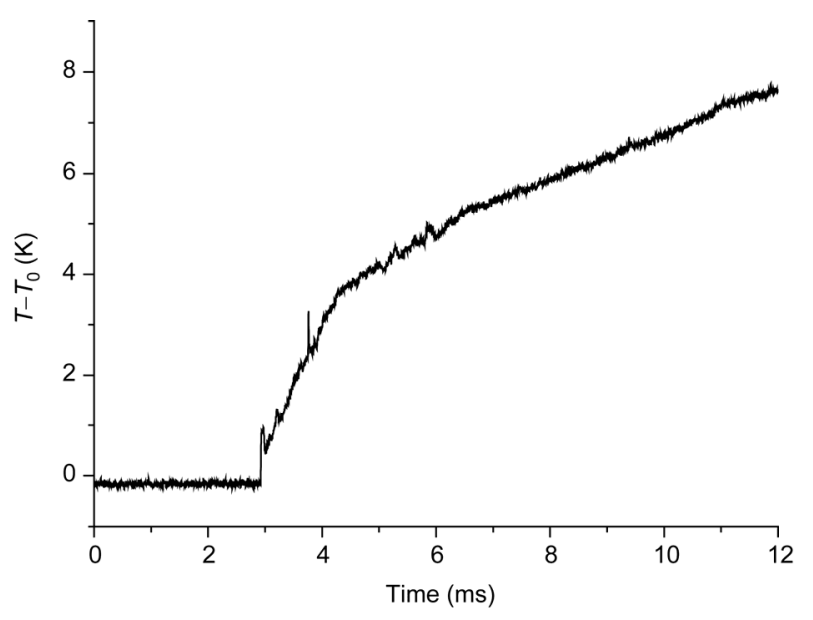

Figure 3 Typical temperature trace for a thermocouple in JF-10.

\section{Test conditions}

Reservoir pressure was measured using piezoelectric pressure transducers mounted on the end of the shock tube. Other reservoir parameters were computed using the measured shock tube filling pressure, shock speed and nozzle reservoir pressure. Based on the reservoir conditions, the freestream was subsequently determined by numerically rebuilding the nozzle flow, a thermo-chemical nonequilibrium program for JF-10 and an equilibrium program for JF-12 [17]. The accuracy of CFD nozzle flow analysis was confirmed through experiments taken in the freestream, including static pressure, pitot pressure and stagnation point heat flux. The reservoir and freestream conditions in these tests are shown in Table 2. The nominal Mach number of JF-10 nozzle is 12 , and 7 for JF-12. Subscript " 0 " represents the reservoir condition and " $\infty$ " for the free-stream condition. Reynolds number, $R e=\frac{\rho_{\infty} u_{\infty} L}{\mu_{\infty}}$, with the characteristic length " $L$ " represents the model length. In addition, the experiments were conducted at surface temperature of 290 $\mathrm{K}$ (room temperature) and zero angle of attack for both JF-10 and JF-12.

\section{Numerical simulations}

As a valuable complement for the analysis of experimental results, such as boundary layer parameter determination, numerical simulations were used in the paper. And calculating heat transfer had also been compared with the experiments. The governing equations employed were axisymmetric, compressible Navier-Stokes equations. Two sets of computation procedure were utilized, taking into account the test conditions of the two shock tunnels and the computing cost. Calorically perfect gas was chosen for the JF-12 flow conditions, while thermal chemical nonequilibrium for the JF-10 conditions and chemical reaction model of air used here was developed by Dunn-Kang, consisted of five species $\left(\mathrm{O}, \mathrm{N}, \mathrm{N}_{2}, \mathrm{O}_{2}, \mathrm{NO}\right)$ without ionization. Beyond that, both procedures were based on the finite difference method of the AUSMPW+ scheme, and point implicit scheme of LU-SGS.

On the solid wall, the no-slip condition for velocity was considered and the temperature was set to the room temperature. The chemical composition on the body surface was either fully catalytic or non-catalytic to chemical reactions. The specific boundary conditions were as below: (1) pressure: $\frac{\partial p}{\partial n}=0$; (2) temperature: $T_{w}=$ constant; (3) velocity: $u=v=0$; (4) components: non-catalytic $\left(\frac{\partial C_{i}}{\partial n}=0\right)$ or fully catalytic $\left(C_{\mathrm{O}_{2}}=0.235, C_{\mathrm{N}_{2}}=0.765\right)$. The subscript " $n$ "

Table 2 Test conditions for JF-10 and JF-12

\begin{tabular}{cccc}
\hline \multicolumn{2}{c}{ Test condition } & JF-10 & JF-12 \\
\hline Reservoir & $P_{0}(\mathrm{MPa})$ & 13.5 & 2.2 \\
& $H_{0}(\mathrm{MJ} / \mathrm{kg})$ & 16 & 3.3 \\
Freestream & $T_{\infty}(\mathrm{K})$ & 435 & 293 \\
& $\rho_{\infty}\left(\mathrm{kg} / \mathrm{m}^{3}\right)$ & $7.0 \times 10^{-4}$ & $5.0 \times 10^{-3}$ \\
& $u_{\infty}(\mathrm{m} / \mathrm{s})$ & 4979 & 2343 \\
& $p_{\infty}(\mathrm{Pa})$ & 95 & 417 \\
$L(\mathrm{~mm})$ & 590 & 1100 \\
& $R e / L(/ \mathrm{m})$ & $1.5 \times 10^{5}$ & $6.5 \times 10^{5}$ \\
& 12 & 7 \\
\hline
\end{tabular}


denoted the normal derivative at the model surface and $C_{i}$ was the mass fraction for species $i$.

The convective heat transfer rate along the model surface was measured under the freestream conditions listed in Table 2. Comparison was made between experimental and numerical values of heat transfer rate. The latter was calculated by combing 3 contributing parts: translational temperature model flux, vibrational temperature model flux and diffusion model flux (only translational temperature model flux was considered for JF-12 condition):

$$
q_{w}=k \frac{\partial T}{\partial n}+k_{v} \frac{\partial T_{v}}{\partial n}+\sum_{i=1}^{n s} h_{i} \rho D_{i} \frac{\partial C_{i}}{\partial n} .
$$

\section{Results and discussion}

High precision heat transfer measurements are relatively difficult to obtain in hypersonic facilities, due to their short test time and harsh environment. Therefore, high precision sensors are a key technology in current measurements. In the paper, the deviation of our home-made thermocouples was first discussed. Stanton number $(S t)$ obtained experimentally and theoretically along the surface of the JF-12 model was shown in Figure 4, where $S t$ was defined in eq. (3) normalized using the freestream parameters,

$$
S t=\frac{q_{w}}{\rho_{\infty} u_{\infty} H_{0}} .
$$

Eq. (3) is usually applied in aerodynamic experiments for what freestream parameters are known in general, but not for the boundary layer parameters. $x$ is the distance along the model to the tip. Because the angle of attack was zero, "Windward" was defined to represent the upper generatrix of the model, "Leeward" for the lower generatrix and "Side" for the horizontal generatrix. Perfect gas was assumed for JF-12 and heat transfer on a sharp cone can be obtained easily by solving boundary layer equations for this model [18], but not for JF-10, where the gas was in thermo-chemical nonequilibrium. Squares, up-triangles and down-triangles in Figure 4 depict heat flux distribution on the side, leeward and windward of the model, respectively, all of which demonstrate good repeatability and uniformity of the freestream flows. Comparison between experimental and theoretical data showed that the experiment was within $\pm 10 \%$ dispersion of the theoretical value, which was mainly coming from the individual differences in thermocouple output voltages due to uneven quality. Overall, the heat transfer measurements obtained in this study were quite good for hypersonic flows.

Heat transfer data obtained at hypersonic flows can also play an important role in code validation, especially for a thermo-chemical nonequilibrium program. In return, CFD readily provides detailed flow field information, such as the boundary layer parameters. Comparison between experimental data and CFD is shown in Figure 5 for both JF-12 and JF-10.

Both catalytic and noncatalytic walls were used under the nonequilibrium conditions of JF-10, as shown in Figure 5(b). In the ground test facilities, recombination of dissociated atoms easily occurred inside the boundary layer due to lower wall temperature and higher pressure than flight conditions [19]. Results showed that the model surface was closer to a catalytic than noncatalytic wall. For both shock tunnels, CFD results were slightly smaller than the experimental data, likely attributable to the possible existent slight roughness in the models that was not thoroughly simulated by CFD. Deviation was within the uncertainty of heat transfer measurements $( \pm 10 \%)$, however, suggesting reasonable agreement between experiment and CFD for both tunnels. Notably, the cell Reynolds number or grid independence was taken into account during computation, though neither was discussed in detail in this paper.

To effectively compare the results gathered under different conditions, such as results from flight and ground tests or from different shock tunnel conditions, it is necessary to reduce the data to a suitable nondimensional form. Heat transfer rate is typically normalized into a Stanton number $(S t)$. Heat transfer, the effect of boundary layer parameters on the wall, is affected by freestream flows, such as shock strength or dissociation. It was thus necessary to normalize the heat transfer using boundary layer parameters rather than freestream flows while comparing experimental data from different ground facilities. Fortunately, boundary layer parameters are easily to be obtained with the help of numerical simulation, but not for the heat flux which is relatively difficult with accurately calculation. Pressure coefficient $\left(C_{p}\right), S t$ and $R e_{x}$ are defined in eq. (4). The subscript "e" represents the boundary layer edge parameters and "w" the wall parameters. $r$ is the recovery factor, where $r=\sqrt{P r}$ for laminar flows [20]. $P r$ is the Prandtl number,

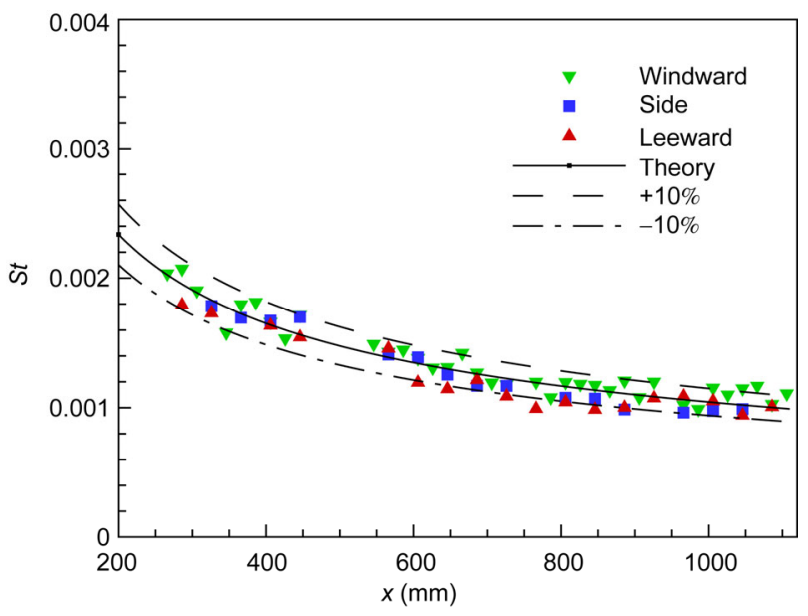

Figure 4 (Color online) Heat transfer data comparison between experiment and theory for the JF-12 shock tunnel model, with St defined in eq. (3). 

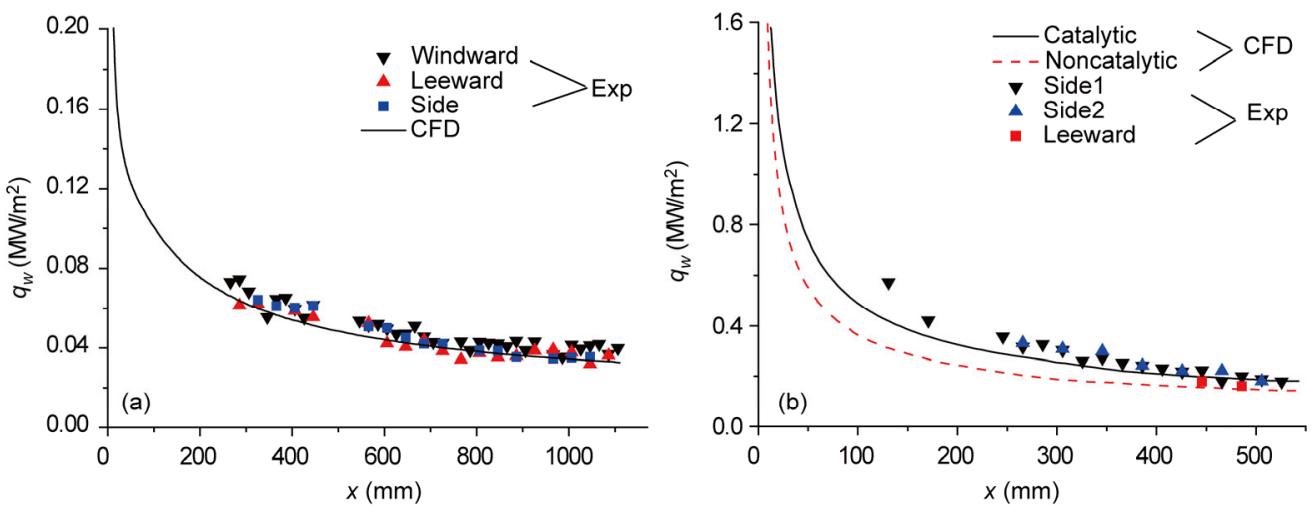

Figure 5 (Color online) Heat transfer data comparison between experiment and CFD in JF-12 and JF-10 shock tunnels. (a) JF-12 condition; (b) JF-10 condition.
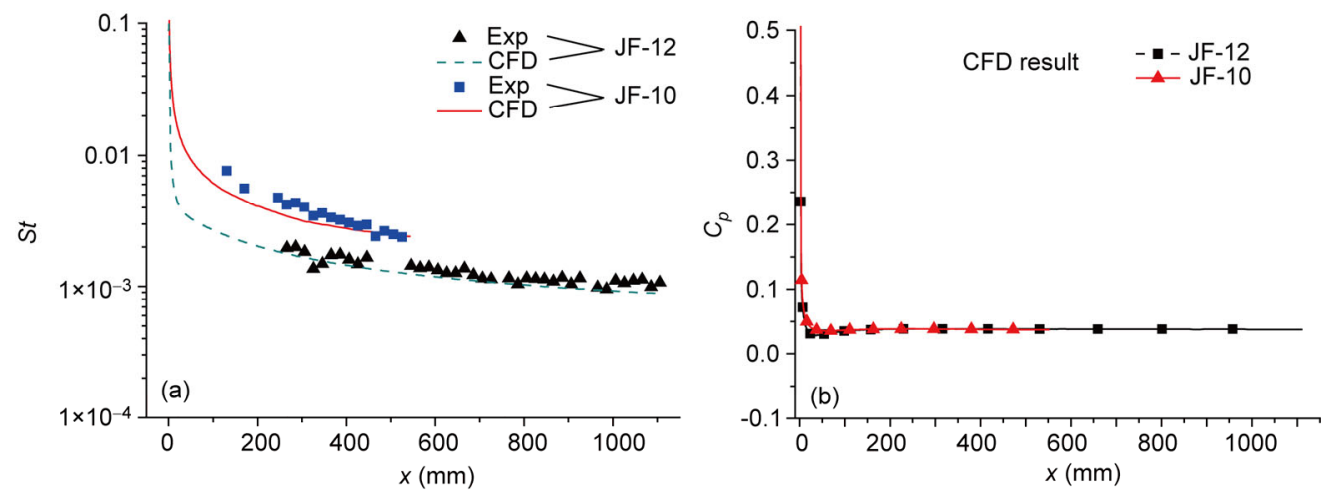

Figure 6 (Color online) Comparison between JF-12 and JF-10 shock tunnels, with St defined in eq. (3). (a) Stanton number; (b) pressure coefficient.

assumed to be constant, a fair approximation under most conditions of interest. Figure 6 shows the comparison including Stanton number and pressure coefficient between JF-12 and JF-10 shock tunnel conditions. Pressure coefficient data was in agreement along the model surface between both tunnels due to the Mach-number independence principle at high Mach numbers, however, the Stanton number didn't eliminate all of the variability caused by differing flow conditions. St in JF-10 was much higher than that in JF-12, indicated by different unit Reynolds numbers. It was thus necessary to select a parameter more representative of these conditions.

$$
\begin{gathered}
S t=\frac{q_{\mathrm{w}}}{\rho_{\mathrm{e}} u_{\mathrm{e}}\left(H_{0}-0.5 \times u_{\mathrm{e}}^{2}(1-r)-H_{\mathrm{w}}\right)}, \\
C_{p}=\frac{p_{\mathrm{w}}-p_{\infty}}{0.5 \rho_{\infty} u_{\infty}^{2}}, R e_{x}=\frac{\rho_{\mathrm{e}} u_{\mathrm{e}} x}{\mu_{\mathrm{e}}} .
\end{gathered}
$$

For an incompressible laminar boundary layer on a flat plate,

$$
S t=\frac{1}{2} C_{f} \operatorname{Pr}^{-2 / 3}, C_{f}=\frac{0.664}{\sqrt{R e_{x}}}
$$

To correct for the compressibility effect, the Eckert reference-temperature concept described by White was used, and the axial symmetry of the sharp cone flow was taken into account by multiplying the result by $\sqrt{3}$ [18]. This gives:

$$
S t=\frac{G\left(M a_{\mathrm{e}}, \operatorname{Pr}, \gamma, T_{\mathrm{w}} / T_{\mathrm{e}}\right)}{\sqrt{\operatorname{Re}_{x}}}=\frac{A}{\sqrt{\operatorname{Re}_{x}}},
$$

where $A$ is a dimensionless constant, the function of $M a_{\mathrm{e}}, P r$, $\gamma, T_{\mathrm{w}} / T_{\mathrm{e}}$. The above equation was derived assuming perfect gas conditions. For the thermo-chemical nonequilibrium condition in JF-10, the effect of air dissociation needed to be taken into account while solving $A$, which is quite difficult theoretically.

Figure 7 shows the Stanton number-Reynolds number relationship for JF-12 and JF-10 shock tunnels, including experimental data, CFD and a fitting line. And it needed to be emphasized that $S t$ and $R e_{x}$ was defined using the boundary layer parameters in eq. (4). Logarithmic coordinates were used to give an intuitive expression of constant $A$. A were 0.66 and 0.67 according to CFD computations for JF-10 and JF-12 respectively, nearly $10 \%$ smaller than the experimental fitting value, almost the same deviation as shown in Figure 5. However, deviation was within the un- 
certainty of heat transfer measurements $( \pm 10 \%)$. Figure 7 also shown that although the Reynolds numbers were quite different from each other, the magnitude of $10^{4}$ for JF-10 and $10^{5}$ for JF-12, the heat transfer along the sharp cone showed the same regularity under parameter $A$, whether or not the flow was in dissociation. Flow chemistry in JF-10 condition had negligible effect on it.

By comparison, Figure 8 showed the Stanton number-Reynolds number relationship using freestream parameters obtained in eq. (3). Parameter $A$ was different for the two shock tunnels while normalized using the freestream flows. The reason was that, heat transfer was the direct effect of boundary layer parameters on the wall. Nonetheless, freestream flows only had indirect effect, where parameters behind the shock were affected by the model configuration or shock strength. Therefore, it is possible to conclude that parameter $A\left(S t \times \sqrt{R e_{x}}\right)$ with $S t, R e_{x}$ defined using bound-

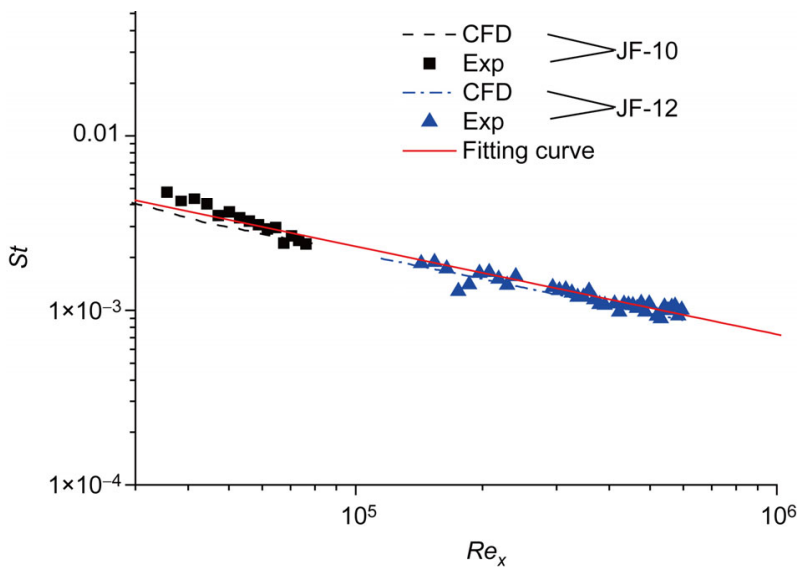

Figure 7 (Color online) Stanton number-Reynolds number relationships for JF-12 and JF-10 models, with $S t, R e_{x}$ defined in eq. (4). Solid line represents a fitting curve for $S t=\frac{0.73}{\sqrt{R e_{x}}}$.

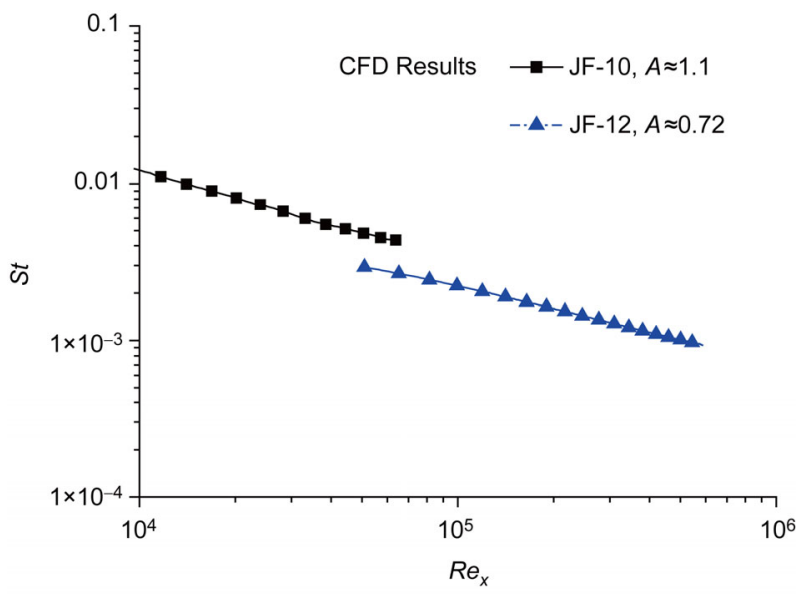

Figure 8 (Color online) Stanton number-Reynolds number relationships for JF-12 and JF-10 models, with $S t, R e_{x}$ defined in eq. (3). ary layer parameters is a more preferable similarity parameter for comparing heat transfer data from two facilities or from ground to flight.

In addition to considering the effect of boundary conditions on $A$, fully catalytic and non-catalytic results under thermo-chemical nonequilibrium condition of JF-10 were also studied in this paper using numerical simulation. Figure 9 shows the Stanton number-Reynolds number relationship obtained in this manner. Although heat transfer rate for the non-catalytic boundary was about $20 \%$ smaller than that for the catalytic boundary, also shown in Figure 5(b), the Stanton numbers agreed well with each other showed the same parameter $A$ of 0.66 . The boundary conditions of whether fully catalytic or non-catalytic had negligible effect on boundary layer edge parameters, but the wall enthalpy $H_{\mathrm{w}}$ in eq. (4) was in difference between almost an order of magnitude. $H_{\mathrm{w}}$ was $0.3 \mathrm{MJ} / \mathrm{kg}$ for catalytic condition and 3 $\mathrm{MJ} / \mathrm{kg}$ for the other. The change of boundary conditions would not only affect the heat transfer, but also the wall parameters, especially $H_{\mathrm{w}}$. Fortunately, eq. (4) can almost eliminate the variability caused by differing boundary conditions and the nondimensional parameter showed the same regularity.

\section{Conclusions}

This study, by building a series of relevant comparisons, investigated hypersonic aerodynamic heating using a sharp cone model configuration in two separate hypersonic facilities at LHD. Based on the experimental and numerical results obtained, several notable conclusions were drawn. First, the scatter difference of heat flux measurements obtained using home-made thermocouples was within $\pm 10 \%$. Parameter $A$, defined as the product of $S t$ and the square root of $R e_{x}$ was proven to be a useful similarity parameter, where Stanton number-Reynolds number relationships was consistent in both shock tunnels. Noted that $S t, \operatorname{Re}_{x}$ should

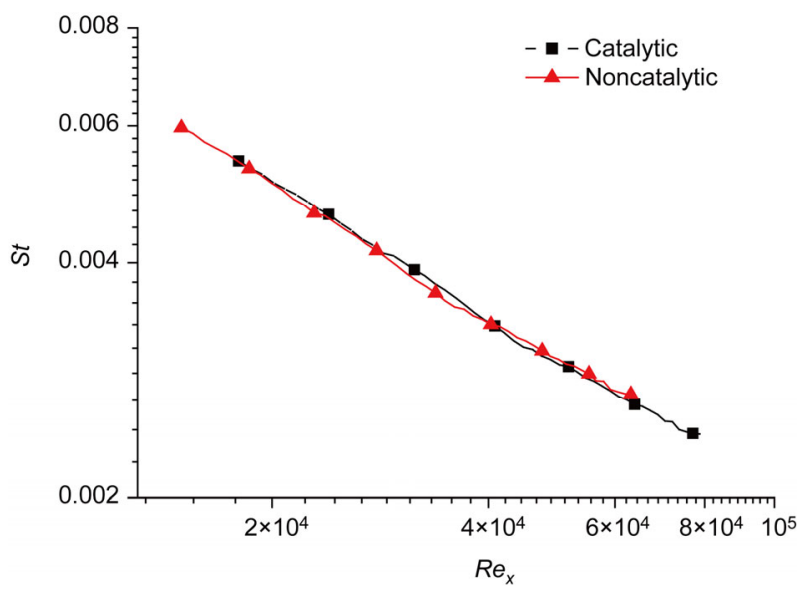

Figure 9 (Color online) Stanton number-Reynolds number relationships for the JF-10 model under catalytic and non-catalytic conditions, with St, $R e_{x}$ defined in eq. (4). 
be normalized using boundary layer parameters, not freestream flow. Finally, parameter $A$ practically eliminates the variability caused by the deferent flow conditions, whether or not the flow is in dissociation or the boundary condition is catalytic. Experiments showed that $A$ is about equal to 0.73 .

This work was supported by the National Natural Science Foundation of China (Grant Nos. 11402275, 11472280 and 11532014).

1 S. L. Gai, and N. R. Nudford, Shock Waves 2, 43 (1992).

2 T. Kishimoto, A. Hanamitsu, and H. Bitoh, in High enthalpy flow computation and experiment around the simple bodies (Special Publication of National Aerospace Laboratory SP-29, Tokyo, 1996) pp. 99.

3 W. Z. Dong, J. L. Le, and T. S. Gao, Exp. Measurem. Fluid Mech. 16, 1 (2002).

4 E. G. Joel, B. J. Heath, and V. C. Graham, J Spacecraft Rockets 51, 455 (2014).

5 C. S. Leonardo, F. Hermann, W. Stefan, and M. Edward, in Numerical investigation of wave-packets in a hypersonic high-enthalpy boundary-layer on a $5^{\circ}$ sharp cone (AIAA, Atlanta, 2014).

6 H. Tanno, T. Komuro,. Sato K, H. Tanno, T. Komuro, K. Sato, K. Itoh, and M. Takahashi, in Measurement of surface pressure fluctuation in hypersonic high-enthalpy boundary layer on a 7-degree cone model (AIAA, Hawaii, 2011).

7 P. Germain, and H. Hornung, The Boundary Layer on A Sharp Cone in High-Enthalpy Flow (Springer, Berlin Heidelberg, 1995), pp. 63-68.
8 X. L. Li, D. X. Fu, and Y. W. Ma, Sci. China-Phys. Mech. Astron. 51, 699 (2008).

9 C. H. Su, and H. Zhou, Sci. China-Phys. Mech. Astron. 52, 1223 (2009).

10 J. B. John, M. Anthony, D. N. Richard, and F. S. Kenneth, in Developing a data base for the calibration and validation of hypersonic CFD codes-sharp cones (AIAA, Orlando, 1993).

11 V. C. Graham, K. S. Pramod, and M. B. Joseph, J. Spacecraft Rockets 52, 17 (2015).

12 H. R. Yu, H. Chen, and W. Zhao, Shock Waves 15, 399 (2006).

13 W. Zhao, Z. L. Jiang, T. Saito, J. M. Lin, H. R. Yu, and K. Takayama, Shock Waves 14, 53 (2004).

14 Z. L. Jiang, and H. R. Yu, in Experiments and development of the long-testduration hypervelocity detonation-driven shock tunnel (LHDst) (AIAA, Maryland, 2014).

15 F. K. Lu, and D. E. Marren, Advanced Hypersonic Test Facilities (Alexander Bell Drive, Reston, 2002).

16 D. L. Schultz, and T. V. Jones, Heat Transfer Measurements in Short Duration Facilities, Technical Report, AGARD-AG-165 (University of Oxford, 1973).

17 Q. Wang, Experimental Study on Characteristics of Heat Transfer and Electron Density in High Enthalpy Flow, Dissertation for the Doctoral Degree (Institute of Mechanics, Chinese Academy of Sciences, Beijing, 2013), pp. 44-50.

18 J. D. Anderson, Hypersonic and High Temperature Gas Dynamics (McGraw-Hill Book Company, New York, 1989).

19 N. R. Mudford, S. L. Gai, and S. G. Mallinson, Boundary layer recombination pathways in bluff body flows in a shock tunnel: Proceedings of 21th ISSW (Panther Pub., Great Keppel, 1997).

20 F. M. White, Viscous Fluid Flow (McGraw-Hill, New York, 1991). 